\title{
Diclofenac Potassium
}

National Cancer Institute

\section{Source}

National Cancer Institute. Diclofenac Potassium. NCI Thesaurus. Code C47983.

The potassium salt form of diclofenac, a benzene acetic acid derivate and nonsteroidal anti-inflammatory drug (NSAID) with analgesic, antipyretic and anti-inflammatory activity. Diclofenac potassium is a non-selective reversible and competitive inhibitor of cyclooxygenase (COX), subsequently blocking the conversion of arachidonic acid into prostaglandin precursors. This leads to an inhibition of the formation of prostaglandins that are involved in pain, inflammation and fever. 